MATHEMATICS OF COMPUTATION

Volume 67, Number 223, July 1998, Pages 1225-1245

S 0025-5718(98)00939-9

\title{
MINUS CLASS GROUPS OF THE FIELDS OF THE $l$-TH ROOTS OF UNITY
}

\author{
RENÉ SCHOOF
}

\begin{abstract}
We show that for any prime number $l>2$ the minus class group of the field of the $l$-th roots of unity $\overline{\mathbf{Q}_{p}}\left(\zeta_{l}\right)$ admits a finite free resolution of length 1 as a module over the ring $\widehat{\mathbf{Z}}[G] /(1+\iota)$. Here $\iota$ denotes complex conjugation in $G=\operatorname{Gal}\left(\overline{\mathbf{Q}_{p}}\left(\zeta_{l}\right) / \overline{\mathbf{Q}_{p}}\right) \cong(\mathbf{Z} / l \mathbf{Z})^{*}$. Moreover, for the primes $l \leq 509$ we show that the minus class group is cyclic as a module over this ring. For these primes we also determine the structure of the minus class group.
\end{abstract}

\section{INTRODUCTION}

Let $l$ be an odd prime and let $\zeta_{l}$ denote a primitive $l$-th root of unity. In this paper we study the cyclotomic fields $\mathbf{Q}\left(\zeta_{l}\right)$ and the class groups $C l_{l}$ of their rings of integers $\mathbf{Z}\left[\zeta_{l}\right]$. The class group $C l_{l}$ splits in a natural way into two parts: the natural map from the class group $C l_{l}^{+}$of the ring of integers of the subfield $\mathbf{Q}\left(\zeta_{l}+\zeta_{l}^{-1}\right)$ to $C l_{l}$ is injective [24, p.40]. Its cokernel, the minus class group of $\mathbf{Q}\left(\zeta_{l}\right)$, is denoted by $C l_{l}^{-}$. There is an exact sequence

$$
0 \longrightarrow \mathrm{Cl}_{l}^{+} \longrightarrow \mathrm{Cl}_{l} \longrightarrow \mathrm{Cl}_{l}^{-} \longrightarrow 0 .
$$

About the groups $\mathrm{Cl}_{l}^{+}$little is known. For small primes $l$ they are trivial [23]. See [3], [21] for a numerical study of these groups. In this paper we consider the other groups, the minus class groups $C l_{l}^{-}$, which are easier to handle. There is, first of all, an explicit and easily computable formula for their cardinalities $h_{l}^{-}$. See $[24$, p.42]:

$$
h_{l}^{-}=2 l \prod_{\chi \text { odd }}-\frac{1}{2} B_{1, \chi}
$$

where the product runs over the characters $\chi:(\mathbf{Z} / l \mathbf{Z})^{*} \longrightarrow \mathbf{C}^{*}$ which are odd, i.e. which satisfy $\chi(-1)=-1$. The numbers $B_{1, \chi}$ are generalized Bernoulli numbers; they are defined in section 1.

Around 1850, E. E. Kummer [9], [10] used this formula to compute the minus class numbers $h_{l}^{-}$for the primes $l<100$. These calculations were extended by D. H. Lehmer and J. M. Masley [15] in 1978 to the primes $l \leq 509$. The numbers $h_{l}^{-}$grow very rapidly with $l$. For instance, $h_{491}^{-}$already has 138 decimal digits.

The class number $h_{l}^{-}$alone does, of course, not determine the structure of the group $C l_{l}^{-}$. If $h_{l}^{-}$is squarefree, the group $C l_{l}^{-}$is cyclic, but in general $h_{l}^{-}$has

Received by the editor March 28, 1994 and, in revised form, December 2, 1996.

1991 Mathematics Subject Classification. Primary 11R18, 11R29, 11R34.

Key words and phrases. Cyclotomic fields, class groups, cohomology of groups. 
multiple factors. It is a natural problem to try and determine the structure of the minus class groups. Kummer [12] addressed this problem in 1853. He showed, for instance, that for $l=29$ the minus class group is isomorphic to $\mathbf{Z} / 2 \mathbf{Z} \times \mathbf{Z} / 2 \mathbf{Z} \times \mathbf{Z} / 2 \mathbf{Z}$. He claimed moreover that the minus class group of $\mathbf{Q}\left(\zeta_{31}\right)$ is cyclic of order 9 . Only in 1870 he gave a rigorous proof of this fact [11]. It involves a lenghty calculation in the field $\mathbf{Q}\left(\zeta_{31}\right)$. His claim that the group $C l_{71}^{-}$is cyclic of order $7^{2} \cdot 79241$ is correct, but has, as far as I know, never been justified previously [6].

In this paper we study the structure of the minus class groups $\mathrm{Cl}_{l}^{-}$as Galois modules. Since complex conjugation $\iota$ acts as -1 on $C l_{l}^{-}$, it is natural to study $\mathrm{Cl}_{l}^{-}$ as a module over the ring $\widehat{\mathbf{Z}}[G] /(1+\iota)$ where $\widehat{\mathbf{Z}}$ denotes the profinite $\operatorname{ring} \underset{\leftarrow}{\lim } \mathbf{Z} / n \mathbf{Z}$ and $G=\operatorname{Gal}\left(\mathbf{Q}\left(\zeta_{l}\right) / \mathbf{Q}\right) \cong(\mathbf{Z} / l \mathbf{Z})^{*}$. We prove the following:

Theorem I. Let $l$ be an odd prime. Then there exist an exact sequence of $\widehat{\mathbf{Z}}[G] /(1+\iota)$-modules

$$
0 \longrightarrow L \stackrel{\Theta}{\longrightarrow} L \longrightarrow C l_{l}^{-} \longrightarrow 0
$$

where $L$ is free of finite rank over $\widehat{\mathbf{Z}}[G] /(1+\iota)$.

Theorem I is an immediate consequence of Theorems 2.2(i) and 3.2(i). For small $l$ we can be more precise:

Theorem II. For $l \leq 509$ one can take $L$ of rank 1 in Theorem I. In other words, the minus class group is isomorphic to $\widehat{\mathbf{Z}}[G] /(1+\iota, \Theta)$ as a $\widehat{\mathbf{Z}}[G] /(1+\iota)$-module. Moreover, for $\Theta$ one can take the modified Stickelberger element introduced in section 1.

Theorem II is proved in section 4. In the course of the proof we determine completely the structure of the minus class groups $C l_{l}^{-}$as abelian groups for $l \leq 509$. As an example we mention $\mathrm{Cl}_{491}^{-}$, which we show to be isomorphic to a product of six cyclic groups:

$\mathbf{Z} / 2 \mathbf{Z} \times \mathbf{Z} / 2 \mathbf{Z} \times \mathbf{Z} / 2 \mathbf{Z} \times \mathbf{Z} / 982 \mathbf{Z} \times \mathbf{Z} / 10802 \mathbf{Z} \times \mathbf{Z} / 18680189262665824155664817 /$ $/ 205804054998786681161963704417938182602575815795883211941228272982586 /$ /25221939971178506931727800584004906Z.

Theorem II probably holds for several other primes $l$, but is definitely not true in general. It does, for instance, not hold for $l=3299$. This follows from the fact that, when $l \equiv 3(\bmod 4)$, the minus class group $C l_{l}^{-}$is cyclic over $\widehat{\mathbf{Z}}[G] /(1+\iota)$ if and only if the class group of the quadratic subfield $\mathbf{Q}(\sqrt{-l}) \subset \mathbf{Q}\left(\zeta_{l}\right)$ is a cyclic group. Since the class group of $\mathbf{Q}(\sqrt{-3299})$ is isomorphic to $\mathbf{Z} / 3 \mathbf{Z} \times \mathbf{Z} / 9 \mathbf{Z}$, the group $C l_{3299}^{-}$is not cyclic as a $\widehat{\mathbf{Z}}[G] /(1+\iota)$-module [13, p.80].

Finally, we single out a particularly simple consequence of our results. Roughly speaking, it says that for prime divisors $p$ of $l-1$, the $p$-part of $C l_{l}^{-}$is cyclic whenever it is small.

Theorem III. Let $l$ and $p$ be odd primes and let $M$ denote the p-part of the minus class group of $\mathbf{Q}\left(\zeta_{l}\right)$. If $\# M$ divides $(l-1)^{2}$, then $M$ is a cyclic group.

Theorem III is proved in section 2. Applying it with $l=31, p=3$ and $l=$ $71, p=7$ respectively we obtain a proof of Kummer's claims. The condition that 
\# $M$ divide $(l-1)^{2}$ cannot be relaxed further: in section 4 we show that the 5 -part of the minus class group of $\mathbf{Q}\left(\zeta_{101}\right)$ is isomorphic to $\mathbf{Z} / 125 \mathbf{Z} \times \mathbf{Z} / 25 \mathbf{Z}$.

Our method is, in some sense, a finite version of Iwasawa theory. It is closely related to V. A. Kolyvagin's work [7]. In order to obtain information about the structure of a certain $\chi$-eigenspace of the $p$-part of a minus class group, we "deform" the Dirichlet character $\chi$ and study the extension $L$ corresponding to $\chi \psi$, where $\psi$ is some character of $p$-power order. The generalized Bernoulli numbers $B_{1, \chi \psi}$ contain information about the $\chi$-eigenspace of the class group of this extension. This information is obtained by viewing the field $L$ as a "truncated" $\mathbf{Z}_{p}$-extension and by studying the $\chi$-part of the minus class group of $L$ by mimicking techniques from Iwasawa theory. The main results are Theorem III and the two criteria for cyclicity, Theorems 2.3 and 3.3 .

The main difficulty in extending Theorem II to primes $l>509$ is the size of the class numbers. For larger $l$ one is bound to encounter composite numbers that cannot be factored within reasonable time. Sooner or later one will also encounter $\chi$-parts that are not cyclic Galois modules. In these cases the methods of this paper do not apply.

The paper is organized as follows. In section 1 we briefly recall some well known facts concerning $\mathbf{Z}[G]$-modules when $G$ is a finite abelian group. In this section we also discuss some elementary properties of Stickelberger elements and generalized Bernoulli numbers. Even though there are similarities between the structure of the odd and even parts of the minus class groups, the differences are sufficiently big to merit separate treatment. In section 2 we consider the $p$-parts of minus class groups for odd primes $p$. In section 3 we do the same for $p=2$. Finally, in section 4 , we present the numerical results and prove Theorem II.

We need to know the complete prime decomposition of the class numbers $h_{l}^{-}$ for $l \leq 509$. In the appendix a table of the prime factorizations of these numbers is given. This table is complete and supersedes the one computed by Lehmer and Masley [15]. The present table contains also the factorizations of the unfactored composite numbers in their table. I thank Arjen Lenstra, Peter Montgomery, Bob Silverman and Herman te Riele for computing the unknown prime factors, François Morain for several primality proofs and Pietro Cornacchia for catching an error in Table 4.4 .

\section{Preliminaries}

In this section we recall some elementary facts concerning modules over group rings $\mathbf{Z}[G]$ when $G$ is a finite abelian group. In addition we recall some basic properties of Stickelberger elements and generalized Bernoulli numbers.

Let $G$ be a finite abelian group. For a $G$-module $M$, we denote by $M^{G}$ the subgroup of $G$-invariant elements of $M$. Now fix a prime $p$ and let

$$
G \cong \pi \times \Delta,
$$

where $\pi$ is the $p$-part of $G$ and $\Delta$ is the maximal subgroup of $G$ of order prime to $p$. We write the group ring $\mathbf{Z}_{p}[G]$ as $\mathbf{Z}_{p}[\Delta][\pi]$. By the orthogonality relations there is an isomorphism of rings

$$
\mathbf{Z}_{p}[\Delta] \cong \prod_{\chi} O_{\chi} .
$$


Here $\chi$ runs over the characters $\chi: \Delta \longrightarrow \overline{\mathbf{Q}}_{p}^{*}$ up to $\operatorname{Gal}\left(\overline{\mathbf{Q}}_{p} / \mathbf{Q}_{p}\right)$-conjugacy. The rings $O_{\chi}$ are unramified extensions of $\mathbf{Z}_{p}$ generated by the values of $\chi$. They are $\mathbf{Z}_{p}[\Delta]$-algebras via the rule $\sigma \cdot x=\chi(\sigma) x$ for $x \in O_{\chi}$ and $\sigma \in \Delta$. The ring isomorphism is given by mapping $\sigma \in \Delta$ to $\chi(\sigma)$ in each component $O_{\chi}$. The residue field of $O_{\chi}$ is $\mathbf{F}_{p}\left(\zeta_{d}\right)$ where $d$ is the order of $\chi$.

Definition. Let $M$ be a $\mathbf{Z}_{p}[G]$-module and let $\chi: \Delta \longrightarrow \overline{\mathbf{Q}}_{p}^{*}$ be a character. Equivalently, $\chi$ is a character of $G$ of order prime to $p$. The $\chi$-eigenspace $M(\chi)$ or $\chi$-part of $M$ is defined by

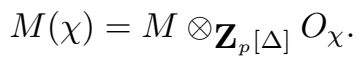

We have a decomposition into eigenspaces of $M$ :

$$
M \cong \prod_{\chi} M(\chi),
$$

where $\chi$ runs over the characters $\chi: \Delta \longrightarrow \overline{\mathbf{Q}}_{p}^{*}$ up to $\operatorname{Gal}\left(\overline{\mathbf{Q}}_{p} / \mathbf{Q}_{p}\right)$-conjugacy. Each eigenspace $M(\chi)$ is a module over the local ring $O_{\chi}[\pi]$. The residue field of this ring is equal to the residue field of $O_{\chi}$ which is $\mathbf{F}_{p}\left(\zeta_{d}\right)$, where $d$ is the order of $\chi$.

We frequently use the following properties of the Tate cohomology groups [2]. Let $M$ be a $G$-module and let $P \subset \pi$. The natural action of $P$ on the Tate cohomology groups $\widehat{H}^{q}(P, M)$ is trivial, but $\Delta$ acts, in general, in a non-trivial way. Note that the groups $\widehat{H}^{q}(P, M)$ are $\mathbf{Z}_{p}[\Delta]$-modules, because they are killed by $\# P$.

Lemma 1.1. Let $p$ be a prime and let $G$ be a finite abelian group. Let $\pi$ and $\Delta$ be as above and let $P$ be a subgroup of $\pi$.

(i) For every $\mathbf{Z}[G]$-module $M$ we have that $\widehat{H}^{q}\left(P, M^{\Delta}\right) \cong \widehat{H}^{q}(P, M)^{\Delta}$ for all $q \in \mathbf{Z}$.

(ii) For every $\mathbf{Z}_{p}[G]$-module $M$ and every character $\chi: \Delta \longrightarrow \overline{\mathbf{Q}}_{p}^{*}$ we have that

$$
\widehat{H}^{q}(P, M(\chi)) \cong \widehat{H}^{q}(P, M)(\chi) \quad \text { for all } q \in \mathbf{Z} .
$$

Proof. (i) Since the actions of $\Delta$ and $P$ commute, the inclusion $i: M^{\Delta} \hookrightarrow M$ and the $\Delta$-norm map $N: M \rightarrow M^{\Delta}$ are $P$-morphisms. The maps $i \cdot N$ and $N \cdot i$ induce multiplication by $\# \Delta$ on $\widehat{H}^{q}(P, M)^{\Delta}$ and $\widehat{H}^{q}\left(P, M^{\Delta}\right)$ respectively. Since $\# \Delta$ and $\# P$ are coprime, multiplication by \# $\Delta$ is an isomorphism and (i) follows.

(ii) Since the actions of $\Delta$ and $P$ commute, the eigenspaces $M(\chi)$ are $P$-modules. Taking the sum over the characters $\chi: \Delta \longrightarrow \overline{\mathbf{Q}}_{p}^{*}$, up to $\operatorname{Gal}\left(\overline{\mathbf{Q}}_{p} / \mathbf{Q}_{p}\right)$-conjugacy, of the natural maps $\widehat{H}^{q}(P, M(\chi)) \longrightarrow \widehat{H}^{q}(P, M)(\chi)$, we obtain precisely the map $\bigoplus_{\chi} \widehat{H}^{q}(P, M(\chi)) \longrightarrow \widehat{H}^{q}(P, M)$ induced by the isomorphism $\bigoplus_{\chi} M(\chi) \longrightarrow M$. This proves (ii).

The remainder of this section is devoted to properties of Stickelberger elements and generalized Bernoulli numbers. Let $f \not \equiv 2(\bmod 4)$ be a conductor and let $G=(\mathbf{Z} / f \mathbf{Z})^{*}$. The Stickelberger element $\theta_{f}$ of conductor $f$ is given by

$$
\theta_{f}=\sum_{\substack{a=1 \\ \operatorname{gcd}(a, f)=1}}^{f}\left(\frac{a}{f}-\frac{1}{2}\right)[a]^{-1} \in \mathbf{Q}[G] .
$$

For any prime number $p$ we write $G=\pi \times \Delta$ as above. We have $\mathbf{Q}_{p}[G] \cong$ $\bigoplus_{\chi} K_{\chi}[\pi]$ where the sum runs over the characters $\chi: \Delta \longrightarrow \overline{\mathbf{Q}}_{p}^{*}$ up to $\operatorname{Gal}\left(\overline{\mathbf{Q}}_{p} / \mathbf{Q}_{p}\right)$ conjugacy and $K_{\chi}$ is the quotient field of $O_{\chi}$. We denote the algebra homomorphism 
$\mathbf{Q}_{p}[G] \longrightarrow K_{\chi}[\pi]$ induced by $\chi$ again by $\chi$. For every character $\chi \neq \omega$, the image $\frac{1}{2} \chi\left(\theta_{f}\right)$ of $\frac{1}{2} \theta_{f}$ in $K_{\chi}[\pi]$ is an element of the subring $O_{\chi}[\pi]$. Here $\omega:(\mathbf{Z} / p \mathbf{Z})^{*} \longrightarrow \overline{\mathbf{Q}}_{p}^{*}$ denotes the Teichmüller character. It is the character that gives the action of $\operatorname{Gal}(\overline{\mathbf{Q}} / \mathbf{Q})$ on the group $\mu_{p}$ of $p$-th roots of unity. Note that $\omega=1$ when $p=2$. For odd $p$ the element $\frac{1}{2} \theta_{f}$ annihilates the $\chi$-part of the $p$-part of the ideal class group of $\mathbf{Q}\left(\zeta_{f}\right)$. This is Stickelberger's Theorem [24, Chpt.6]. For $p=2$, C. Greither [4] has shown the same when $\pi$ is cyclic and the conductor $f$ is odd.

For any character $\varphi$ of $G$ of conductor $f$, the generalized Bernoulli number $B_{1, \varphi}$ is simply the value of the algebra homomorphism $\mathbf{Q}_{p}[G] \longrightarrow \overline{\mathbf{Q}}_{p}$ induced by $\varphi$ evaluated on the Stickelberger element:

$$
B_{1, \varphi}=\varphi\left(\theta_{f}\right)=\sum_{\substack{a=1 \\ \operatorname{gcd}(a, f)=1}}^{f}\left(\frac{a}{f}-\frac{1}{2}\right) \varphi(a)^{-1} \in \overline{\mathbf{Q}}_{p} .
$$

Finally we assume that $f=l$ is prime, so that $G=(\mathbf{Z} / l \mathbf{Z})^{*}$ and we introduce the modified Stickelberger element $\Theta \in \widehat{\mathbf{Z}}[G] /(1+\iota)$ that occurs in Theorem II. We have that $\widehat{\mathbf{Z}}[G] /(1+\iota) \cong \prod_{p} \mathbf{Z}_{p}[G] /(1+\iota)$. Moreover, each factor $\mathbf{Z}_{p}[G] /(1+\iota)$ is isomorphic to $\prod_{\chi} O_{\chi}\left[\pi_{p}\right]$, where the $\chi$ run over all odd characters of order prime to $p$ when $p$ is odd and all characters of odd order when $p=2$ respectively. Here $\pi_{p}$ denotes the $p$-part of $G$. Therefore it suffices to describe the various components $\chi(\Theta)$ of $\Theta$ : if $p=l$ and $\chi=\omega$ or if $p=2$ and $\chi=1$, we let $\chi(\Theta)=1$. In all other cases $\chi(\Theta)=\frac{1}{2} \chi\left(\theta_{l}\right)$.

The modified Stickelberger element $\Theta \in \widehat{\mathbf{Z}}[G](1+\iota)$ annihilates $C l_{l}^{-}$. The order of $\widehat{\mathbf{Z}}[G](1+\iota, \Theta)$ is equal to the minus class number $h_{l}^{-}$.

\section{ODD PRIMES $p$}

In this section we study the $p$-parts of the minus class groups of complex abelian number fields for odd primes $p$. We show that certain eigenspaces of these groups are cohomologically trivial Galois modules. This puts restraints on their structure. We derive an easily applicable criterion for these eigenspaces to be cyclic Galois modules.

In this section $p \neq 2$ is a prime. We fix a complex abelian number $K$ field with $G=\operatorname{Gal}(K / \mathbf{Q})$. Let $\pi$ denote the $p$-part of $G$ and $F=K^{\pi}$ its fixed field. We fix an odd character $\chi: G \longrightarrow \overline{\mathbf{Q}}_{p}{ }^{*}$ of order prime to $p$, which is not equal to the Teichmüller character $\omega$. Since $p \neq 2$, we have that $C l_{K}^{-}(\chi)=C l_{K}(\chi)$. Therefore we work, in this section, with the class group $C l_{K}$ itself rather than the minus class group $C l_{K}^{-}$.

Theorem 2.1. Let $P \subset G$ be a subgroup of $\pi$ with fixed field $E=K^{P}$. Suppose that that for all primes $r$ that are ramified in $E \subset K$ we have that $\chi(r) \neq 1$. Then

(i) the eigenspace $C l_{K}(\chi)$ is a cohomologically trivial $O_{\chi}[P]$-module;

(ii) the natural map $C l_{E}(\chi) \longrightarrow C l_{K}(\chi)^{P}$ is bijective and the norm $\operatorname{map}_{C} C l_{K}(\chi)$ $\longrightarrow C l_{E}(\chi)$ is surjective.

Proof. (i) It suffices to show that $\widehat{H}^{q}\left(P, C l_{K}(\chi)\right)=0$ for all $q \in \mathbf{Z}$. Let $O_{K}$ denote the ring of integers of $K$, let $C_{K}$ denote the idèle class group of $K$ and let $U_{K}$ denote the group of unit idèles, i.e. the group of $K$-idèles that have trivial valuation at all 
finite primes. We have the exact sequence of $G$-modules [2]

$$
0 \longrightarrow O_{K}^{*} \longrightarrow U_{K} \longrightarrow C_{K} \longrightarrow l_{K} \longrightarrow 0 .
$$

We show that the $\chi$-parts of the Tate $P$-cohomology groups of these modules are all zero. For the unit group $O_{K}^{*}$ we have the following exact sequence $[24$, p.39]

$$
0 \longrightarrow\{1,-1\} \longrightarrow \mu_{K} \times O_{K^{+}}^{*} \longrightarrow O_{K}^{*} \longrightarrow Q \longrightarrow 0 .
$$

Here $O_{K^{+}}$is the ring of integers of the maximal real subfield $K^{+}$of $K$ and $\mu_{K}$ denotes the group of roots of unity in $K$. The group $Q$ has order at most 2 . Complex conjugation acts trivially on $\{1,-1\}$, on $Q$ and on $O_{K^{+}}^{*}$. Since $\chi$ is an odd character, we have, by Lemma 1.1 , that $\widehat{H}^{q}\left(P, O_{K}^{*}\right)(\chi) \cong \widehat{H}^{q}\left(K, \mu_{K}\right)(\chi)$ for all $q \in \mathbf{Z}$. Since $\chi$ is not the Teichmüller character, the $\chi$-part of $\mu_{K}$ is zero so that, by Lemma $1.1, \widehat{H}^{q}\left(P, O_{K}^{*}\right)(\chi)=0$ for all $q \in \mathbf{Z}$.

By global class field theory there are natural isomorphisms $\widehat{H}^{q}\left(P, C_{K}\right) \cong$ $\widehat{H}^{q-2}(P, \mathbf{Z})$ for all $q \in \mathbf{Z}$. Since $G$ acts trivially on $\mathbf{Z}$, it follows from Lemma 1.1 that $\widehat{H}^{q}\left(P, C_{K}\right)(\chi)=0$ for all $q \in \mathbf{Z}$.

We use local class field theory to compute the cohomology of $U_{K}$. See also [20]. By Shapiro's lemma we have

$$
\widehat{H}^{q}\left(P, U_{K}\right) \cong \bigoplus_{v} \widehat{H}^{q}\left(P_{r}, O_{w}^{*}\right)=\bigoplus_{r} \bigoplus_{v \mid r} \widehat{H}^{q}\left(P_{r}, O_{w}^{*}\right)
$$

where $v$ runs over the prime ideals of $E$ and $r$ runs over ordinary prime numbers. The ring $O_{w}$ is the ring of integers of the completion $K_{w}$ of $K$ at a prime $w$ of $K$ over $v$. We have $\mathbf{Q}_{r} \subset E_{v} \subset K_{w}$ with Galois groups $G_{r}=\operatorname{Gal}\left(K_{w} / \mathbf{Q}_{r}\right)$, $P_{r}=\operatorname{Gal}\left(K_{w} / E_{v}\right)$ and $H_{r}=\operatorname{Gal}\left(E_{v} / \mathbf{Q}_{r}\right)$. Since $G$ is abelian, the decomposition groups $P_{r}$ and $H_{r}$ only depend on the prime $r$. Since $\widehat{H}^{q}\left(P_{r}, O_{w}^{*}\right)$ vanishes when $v$ is unramified in $K$, it suffices to consider only primes $r$ that are ramified in $E \subset K$. For each prime ideal $v$ of $F$ dividing a ramified prime $r$, there is an exact sequence of $G_{r}$-modules

$$
0 \longrightarrow O_{w}^{*} \longrightarrow K_{w}^{*} \longrightarrow \mathbf{Z} \longrightarrow 0 .
$$

Consider the long exact sequence of Tate $P_{r}$-cohomology groups. By Lemma 1.1, the group $H_{r}$ acts trivially on the cohomology groups $\widehat{H}^{q}\left(P_{r}, \mathbf{Z}\right)$. By local class field theory there are natural isomorphisms $\widehat{H}^{q}\left(P_{r}, K_{w}^{*}\right) \cong \widehat{H}^{q-2}\left(P_{r}, \mathbf{Z}\right)$ for all $q \in \mathbf{Z}$, so that $H_{r}$ also acts trivially on the groups $\widehat{H}^{q}\left(P_{r}, K_{w}^{*}\right)$. Let $\Delta_{r}$ denote the maximal subgroup of $H_{r}$ of order prime to $p$. Then $\Delta_{r}$ and $P_{r}$ have coprime orders, so that the long cohomology sequence remains exact when we take $\Delta_{r}$-invariants. It follows that $\widehat{H}^{q}\left(P_{r}, O_{w}^{*}\right)$ is $\Delta_{r}$-invariant. Therefore $\Delta_{r}$ acts trivially on the sum $\bigoplus_{v \mid r} \widehat{H}^{q}\left(P_{r}, O_{w}^{*}\right)$. Since $\chi(r) \neq 1$ for all ramified primes $r$, we see that $\Delta_{r} \not \subset \operatorname{ker}(\chi)$. This implies that the $\chi$-part of $\bigoplus_{v \mid r} \widehat{H}^{q}\left(P_{r}, O_{w}^{*}\right)$ is zero.

It follows that $\widehat{H}^{q}\left(G, U_{K}\right)(\chi)=0$ for all $q \in \mathbf{Z}$. Combining all this and using Lemma 1.1 one more time, we deduce that $\widehat{H}^{q}\left(P, C l_{K}(\chi)\right)=0$ for all $q \in \mathbf{Z}$. This proves (i).

(ii) It is easy to see that the natural map $C_{E} / N\left(C_{K}\right) \longrightarrow C l_{E} / N\left(C l_{K}\right)$ is surjective. Since $\chi \neq 1$, the group $C_{E} / N\left(C_{K}\right)=\widehat{H}^{0}\left(P, C_{K}\right) \cong \widehat{H}^{-2}(P, \mathbf{Z})$ has trivial $\chi$-part, and it follows that the norm map $N: C l_{K}(\chi) \longrightarrow C l_{E}(\chi)$ is surjective. Notice that in order to prove surjectivity of this norm map we have not really used the condition on $\chi$, but merely the fact that $\chi$ is not trivial. 
The $P$-cohomology groups of each module in the exact sequence $0 \longrightarrow O_{K}^{*} \longrightarrow$ $U_{K} \longrightarrow C_{K} \longrightarrow C l_{K} \longrightarrow 0$ have trivial $\chi$-parts. Since the natural maps $O_{E}^{*} \rightarrow$ $O_{K}^{*}{ }^{P}, U_{E} \rightarrow U_{K}^{P}$ and $C_{E} \rightarrow C_{K}^{P}$ are all isomorphisms, so is $C l_{E}(\chi) \rightarrow C l_{K}(\chi)^{P}$. This proves (ii).

Theorem 2.2. If for all primes $r$ that are ramified in $F \subset K$ we have that $\chi(r) \neq$ 1 , then

(i) there is an exact sequence of $O_{\chi}[\pi]$-modules

$$
0 \longrightarrow O_{\chi}[\pi]^{d} \stackrel{\Theta}{\longrightarrow} O_{\chi}[\pi]^{d} \longrightarrow C l_{K}(\chi) \longrightarrow 0
$$

where $d$ is the $O_{\chi}$-rank of $C l_{F}(\chi)$;

(ii) we have

$$
\# C l_{K}(\chi)=\# O_{\chi} /\left(\prod_{\psi} B_{1, \chi^{-1} \psi}\right)
$$

where $\psi$ runs over all characters $\psi: \pi \longrightarrow \overline{\mathbf{Q}}_{p}{ }^{*}$.

Proof. By Nakayama's lemma there is a surjective $O_{\chi}[\pi]$ morphism $O_{\chi}[\pi]^{d} \longrightarrow$ $C l_{K}(\chi)$. By Theorem 2.1, the class group $C l_{K}(\chi)$ and hence the kernel of this map are cohomologically trivial. Now one copies the proof of $[2, \mathrm{p} .113$, Thm.8] with $\mathbf{Z}$ replaced by the discrete valuation ring $O_{\chi}$. It follows that the kernel is a projective $O_{\chi}[\pi]$-module. Since $O_{\chi}[\pi]$ is local, the kernel is therefore free. It has rank $d$ since it is of finite index in $O_{\chi}[\pi]^{d}$. This proves (i).

Part (ii) is a generalization of the Theorem of B. Mazur and A. Wiles [7], [16], [17], [18]. By D. Solomon's Theorem [22, p.472], we have for every subgroup $P \subset \pi$ with cyclic quotient $\pi / P$,

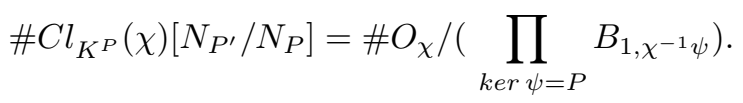

Here the $\psi$ run over the characters of $G$ for which $\operatorname{ker} \psi=P$. Here $P^{\prime}$ denotes the unique subgroup of $\pi$ containing $P$ as a subgroup of index $p$ and $N_{P}$ and $N_{P^{\prime}}$ denote the norm maps $\sum_{\sigma \in P} \sigma$ and $\sum_{\sigma \in P^{\prime}} \sigma$ respectively. In the exceptional case $P=\pi$ the group $P^{\prime}$ is not defined and we simply put $N_{P^{\prime}}=0$. By $C l_{K}^{P}\left[N_{P^{\prime}} / N_{P}\right]$ we denote the kernel of the relative norm map $N_{P^{\prime}} / N_{P}$ from the class group $C l_{K^{P}}(\chi)$ to itself.

Put $S_{\chi}=\prod_{P} N_{P} O_{\chi}[\pi] / N_{P^{\prime}} O_{\chi}[\pi]$. Here $P$ runs over the subgroups of $\pi$ with cyclic quotient $\pi / P$. The natural map

$$
g: O_{\chi}[\pi] \longrightarrow S_{\chi}
$$

becomes an isomorphism when we take the tensor product with the quotient field $K_{\chi}$ of $O_{\chi}$. Therefore $g$ is injective and has finite cokernel.

All modules occurring in the exact sequence of part (i) are cohomologically trivial. Therefore it remains exact when we apply the functor $\prod_{P} N_{P}(-) / N_{P^{\prime}}(-)$ to it. We obtain the following diagram with exact rows.

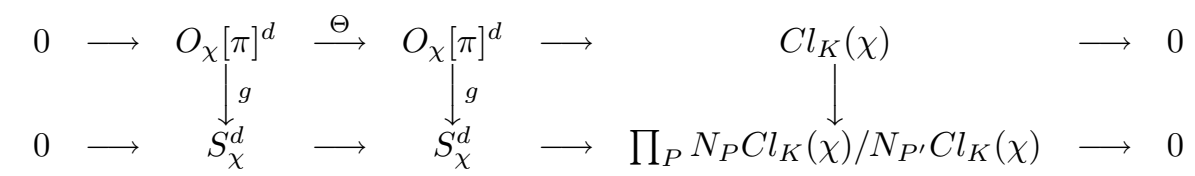


Theorem 2.1(i) and (ii) and an application of the snake lemma then gives that

$$
\# C l_{K}(\chi)=\prod_{P} \#\left(N_{P} C l_{K}(\chi) / N_{P^{\prime}} C l_{K}(\chi)\right)=\prod_{P} \#\left(C l_{K^{P}}(\chi)\left[N_{P^{\prime}} / N_{P}\right]\right)
$$

and the result follows from Solomon's Theorem.

It is not difficult to express the order of $C l_{K}(\chi)$ in terms of the matrix $\Theta$ of Theorem 2.1(i). One has [1, III, sect.9, Prop.6]

$$
\# C l_{K}(\chi)=\# O_{\chi} /\left(\prod_{\psi} \psi(\operatorname{det}(\Theta))\right) .
$$

Here $\psi$ runs over the characters of $\pi$, and $\psi(\operatorname{det}(\Theta))$ indicates the value of the natural extension of $\psi$ to an algebra homomorphism $O_{\chi}[\pi] \longrightarrow \overline{\mathbf{Q}}_{p}$ on $\operatorname{det}(\Theta) \in$ $O_{\chi}[\pi]$.

Next we deduce a sufficient condition for the eigenspace $C l_{K}(\chi)$ to be a cyclic $O_{\chi}[\pi]$-module.

Theorem 2.3. Suppose that for all primes $r$ that are ramified in $F \subset K$ we have that $\chi(r) \neq 1$. If one of the following conditions holds:

$-B_{1, \chi^{-1}}=$ pu for some unit $u \in O_{\chi}^{*}$;

- there exists a character $\varphi: \operatorname{Gal}(\overline{\mathbf{Q}} / \mathbf{Q}) \longrightarrow \overline{\mathbf{Q}}_{p}{ }^{*}$ of order $p^{k}>1$ such that $B_{1, \chi^{-1} \varphi}=\left(1-\zeta_{p^{k}}\right) u$ for some unit $u$ in $O_{\chi}\left[\zeta_{p^{k}}\right]$,

then there is an isomorphism of $O_{\chi}[\pi]$-modules

$$
C l_{K}(\chi) \cong O_{\chi}[\pi] /\left(\theta_{\chi}\right) .
$$

In particular, $\mathrm{Cl}_{K}(\chi)$ is a cyclic $O_{\chi}[\pi]$-module.

Proof. We first show that $C l_{F}(\chi)$ is a cyclic $O_{\chi}$-module. If $B_{1, \chi^{-1}}=p u$ for some unit $u \in O_{\chi}^{*}$, it follows from Theorem 2.2(ii) that $\# C l_{F}(\chi)$ is equal to the order of the residue field $O_{\chi} /(p)$. Therefore $C l_{F}(\chi)$ is cyclic over $O_{\chi}$.

In the other case, let $E=\overline{\mathbf{Q}}^{\operatorname{ker} \varphi} F$ and let $P=\operatorname{Gal}(E / F)$. Then $P$ is cyclic and we let $F \subset E^{\prime} \subset E$ be the unique subfield of $E$ of index $p$. Since $\varphi \neq 1$, it follows from Theorem 2.1(ii) that the norm map $N_{E / E^{\prime}}: C l_{E}(\chi) \longrightarrow C l_{E^{\prime}}(\chi)$ is surjective. To compute the order of the kernel of $N_{E / E^{\prime}}$, we observe that

$$
\operatorname{Norm}\left(B_{1, \chi^{-1} \varphi}\right)=\operatorname{Norm}\left(1-\zeta_{p^{k}}\right)=p
$$

(here the Norm is the $\mathbf{Q}_{p}\left(\zeta_{p^{k}}\right) / \mathbf{Q}_{p^{-}}$norm). By Solomon's Theorem [22, Thm. II, 1], we conclude that $C l_{E}(\chi)\left[N_{E / E^{\prime}}\right]$ has the same order as the residue field $O_{\chi} /(p)$ of $R_{\chi}$. Therefore so does $C l_{E}(\chi) /\left(N_{E / E^{\prime}}\right)$. By Nakayama's lemma, $C l_{E}(\chi)$ is therefore cyclic over the group ring $O_{\chi}[P]$. It follows that $C l_{F}(\chi)$ is cyclic over $O_{\chi}$ in this case as well.

To complete the proof, we observe that, by Theorem 2.1, $C l_{K}(\chi)$ is cohomologically trivial and the $\pi$-norm map induces an $O_{\chi}$-isomorphism between $C l_{F}(\chi)$ and $C l_{K}(\chi)$ modulo the augmentation ideal of $O_{\chi}[\pi]$. It follows from Nakayama's lemma that $C l_{K}(\chi)$ is cyclic over $O_{\chi}[\pi]$. By Stickelberger's theorem there is therefore a surjection $O_{\chi}[\pi] /\left(\theta_{\chi}\right) \longrightarrow C l_{K}(\chi)$, which is an isomorphism because both groups have the same order by Theorem 2.2. This proves Theorem 2.3. 
In the case the $p$-group $\pi$ is cyclic of order $p^{e}$, say, we can be a little bit more explicit. We have the usual isomorphism of local rings, familiar in Iwasawa theory

$$
O_{\chi}[\pi] \cong O_{\chi}[T] /\left((1+T)^{p^{e}}-1\right),
$$

where $1+T$ corresponds to some generator of $\pi$. The maximal ideal of this local ring is $(T, p)$. For $i \geq 0$, we let $\omega_{i}(T)=(1+T)^{p^{i}}-1$.

By the Weierstrass Preparation theorem [24], every non-zero $f(T) \in O_{\chi}[[T]] /$ $\left((1+T)^{p^{e}}-1\right)$ is the residue class of a polynomial of the form $p^{\mu} u(T) h(T)$ where $\mu$ is a non-negative integer, $u(T)$ a unit and $h(T)=T^{\lambda}+a_{\lambda-1} T^{\lambda-1}+\ldots+a_{1} T+a_{0}$ is a Weierstrass polynomial of degree $\lambda<p^{e}$. This means that $a_{i} \equiv 0(\bmod p)$ for $i=0,1, \ldots, \lambda-1$.

Proposition 2.4. Suppose that for all primes $r$ that are ramified in $F \subset K$ we have that $\chi(r) \neq 1$. Suppose that the Galois group $\pi$ is cyclic of order $p^{e}$ and that $C l_{F}(\chi)$ is a cyclic $O_{\chi}$-module. If for some character $\psi$ of $\pi$ of order $p$, for some $\lambda<p-1$ and for some unit $u \in O_{\chi}\left[\zeta_{p}\right]$, we have that $B_{1, \chi^{-1} \psi}=\left(1-\zeta_{p}\right)^{\lambda} u$, then

$$
C l_{K}(\chi) \cong\left(O_{\chi} /\left(p^{e}\right)\right)^{\lambda-1} \times O_{\chi} /\left(p^{e} B_{1, \chi^{-1}}\right)
$$

as an $O_{\chi}$-module.

Proof. We write $O_{\chi}[\pi]=O_{\chi}[T] /\left(\omega_{e}(T)\right)$ as above. Since $C l_{F}(\chi)$ is a cyclic $O_{\chi^{-}}$ module, it follows from Theorem 2.1 that the eigenspace $C l_{K}(\chi)$ is a cohomologically trivial cyclic $O_{\chi}[\pi]$-module. Therefore $C l_{K}(\chi) \cong O_{\chi}[\pi] /\left(p^{\mu} f(T)\right)$ for some Weierstrass polynomial $f(T)$. Since $C l_{F}(\chi) \cong O_{\chi}[\pi] /(T) \cong O_{\chi} /\left(p^{\mu} f(0)\right)$, we have that $p^{\mu} f(0)=B_{1, \chi^{-1}}$, up to a $p$-adic unit. Similarly, for the subfield $F \subset E \subset K$ of degree $p$ over $F$ we have that $C l_{E} \cong O_{\chi}[T] /\left(f(T), \omega_{1}(T)\right)$. Applying Solomon's Theorem [22, Thm. II, 1], we find that, up to a $p$-adic unit, $f\left(1-\zeta_{p}\right)=B_{1, \chi^{-1} \psi}=\left(1-\zeta_{p}\right)^{\lambda}$.

Since $\lambda<p-1$, this implies $\mu=0$ and $\operatorname{deg}(f)=\lambda$. Since $O_{\chi}[T] /\left(f(T), \omega_{e}(T)\right)$ is cohomologically trivial, we have the following exact sequence

$$
0 \longrightarrow O_{\chi}[T] /\left(f(T), \omega_{e}(T) / T\right) \stackrel{T}{\longrightarrow} O_{\chi}[T] /\left(f(T), \omega_{e}(T)\right) \longrightarrow O_{\chi} /(f(0)) \longrightarrow 0 .
$$

We analyze the ideal $\left(f(T), \omega_{e}(T) / T\right)$. Consider for $0 \leq i<e$ the quotient

$$
\frac{\omega_{i+1}(T)}{\omega_{i}(T)}=(1+T)^{p^{i}(p-1)}+\ldots+(1+T)^{p^{i}}+1 .
$$

Since $\lambda<p-1$ we have that $T^{p-1} \equiv T p g(T)(\bmod f(T))$ for some polynomial $g(T) \in O_{\chi}[T]$. This implies that $\omega_{i+1} / \omega_{i}=p+p T h(T)$ for some $h(T) \in O_{\chi}[T]$. Therefore

$$
\frac{\omega_{e}(T)}{T}=\prod_{i=0}^{e-1} \frac{\omega_{i+1}}{\omega_{i}} \equiv p^{e} \cdot u(T)(\bmod f(T))
$$

where $u(T)$ is some unit in $O_{\chi}[T] /\left(\omega_{e}(T)\right)$. This shows that the ideals $(f(T)$, $\left.\omega_{e}(T) / T\right)$ and $\left(f(T), p^{e}\right)$ are equal and that there is an isomorphism of $O_{\chi}$-modules

$$
O_{\chi}[T] /\left(f(T), \omega_{e}(T) / T\right) \cong\left(O_{\chi} / p^{e} O_{\chi}\right)^{\lambda} .
$$

To complete the proof, we observe that $f(0) \in O_{\chi}[T] /\left(f(T), \omega_{e}(T)\right)$ is the image of

$$
\frac{f(T)-f(0)}{T} \in O_{\chi}[T] /\left(f(T), \omega_{e}(T) / T\right)=O_{\chi}[T] /\left(f(T), p^{e}\right),
$$


under the multiplication by $T$ map. Since $f$ is monic, this implies that $f(0)$ has order $p^{e}$. Therefore $1 \in O_{\chi}[T] /\left(\omega_{e}(T), f(T)\right)$ has, up to $p$-adic unit, order $f(0) p^{e}$.

This completes the proof

The following simple result often suffices to determine the structure of the $p$-part of the minus class group of $\mathbf{Q}\left(\zeta_{l}\right)$ when $p$ divides $l-1$. Note that the proof does not rely on the theorems of Mazur-Wiles, Kolyvagin or Solomon.

Theorem III. Let $l$ and $p$ be odd primes and let $M$ be the p-part of the minus class group of $\mathbf{Q}\left(\zeta_{l}\right)$. If $\# M$ divides $(l-1)^{2}$, then $M$ is a cyclic group.

Proof. Let $\pi$ denote the $p$-part of $G=\operatorname{Gal}\left(\mathbf{Q}\left(\zeta_{l}\right) / \mathbf{Q}\right)$; it is a cyclic group of order $p^{e}$. Let $F$ be the fixed field of $\pi$, let $\chi$ be a character of $G$ of order prime to $p$ and let $M(\chi)$ be the corresponding eigenspace of $M$. We assume that $M(\chi) \neq 0$. Since the condition of Theorem 2.1 is satisfied for $K=\mathbf{Q}\left(\zeta_{l}\right)$, there is an exact sequence

$$
0 \longrightarrow O_{\chi}[\pi]^{d} \stackrel{\Theta}{\longrightarrow} O_{\chi}[\pi]^{d} \longrightarrow M(\chi) \longrightarrow 0,
$$

where $d$ is the $O_{\chi}$-rank of $C l_{F}(\chi)$. Let $q=p^{a}$ denote the number of elements in the residue field of $O_{\chi}$. We write $\operatorname{det}(\Theta)=p^{\mu} u(T) f(T) \in O_{\chi}[\pi] \cong O_{\chi}[T] /\left(\omega_{e}(T)\right)$ for some Weierstrass polynomial $f(T)=T^{\lambda}+a_{\lambda-1} T^{\lambda-1}+\ldots+a_{1} T+a_{0}$ and some unit $u(T)$. Then $\# M(\chi)=\# O_{\chi} /\left(\prod_{\zeta^{p^{e}}=1} p^{\mu} f(\zeta-1)\right)$, so that

$$
\# M(\chi) \geq q^{\mu p^{e}+\min (\lambda, p-1) e+1}
$$

and hence

$$
2 e \geq a\left(\mu p^{e}+\min (\lambda, p-1) e+1\right) .
$$

Since $2 e<p^{e}+1$, we have $\mu=0$. Since $M(\chi) \neq 0$, this implies that $\lambda>0$. Moreover, since $a \cdot \min (\lambda, p-1)<2$, we have that $\lambda=1$ and $a=1$ so that $O_{\chi}=\mathbf{Z}_{p}$. This shows that, up to a unit, $f(T)=\operatorname{det}(\Theta)=T-\beta$ for some $\beta \in p \mathbf{Z}_{p}$. Since $d$ is the $O_{\chi}$-rank of $C l_{F}(\chi)$, any surjection $O_{\chi}[\pi]^{d} \longrightarrow C l_{l}(\chi)$ is an isomorphism modulo the maximal ideal $\mathfrak{m}$ of the local $\operatorname{ring} O_{\chi}[\pi]$. This implies that all entries of the matrix $\Theta$ are contained in $\mathfrak{m}$ so that $\operatorname{det}(\Theta) \in \mathfrak{m}^{d}$.

It follows that $d=1$, so that $M(\chi) \cong \mathbf{Z}_{p}[T] /\left((1+T)^{p^{e}}-1, T-\beta\right) \cong \mathbf{Z}_{p} / p^{e} \beta \mathbf{Z}_{p}$ is a cyclic group. We conclude the proof by observing that $\# M(\chi) \geq p^{e+1}$, so that only one eigenspace $M(\chi)$ is non-trivial and hence $M=M(\chi)$.

\section{ThE 2-PART}

In this section we study the 2-part of the minus class group of a complex abelian number field $K$. We show that certain eigenspaces of the 2-part are cohomologically trivial Galois modules. This has consequences for their structure. Finally we prove a criterion for cyclicity of these eigenspaces as Galois modules.

Let $G=\operatorname{Gal}(K / \mathbf{Q})$, let $\iota \in G$ denote complex conjugation and let $K^{+}$denote the fixed field of $\iota$. We have inclusions of idèle class groups $C_{K^{+}} \subset C_{K}$ and of idèle unit groups $U_{K^{+}} \subset U_{K}$. There is a natural map $C l_{K^{+}} \longrightarrow C l_{K}$. We define

$$
\begin{aligned}
U_{K}^{-} & =U_{K} / U_{K^{+}}, \\
C_{K}^{-} & =C_{K} / C_{K^{+}}, \\
C l_{K}^{-} & =C l_{K} / \operatorname{im} C l_{K^{+}}, \\
\mu_{K}^{-} & =\mu_{K} \cap U_{K}^{-} .
\end{aligned}
$$


Note that $U_{K}^{-}$is isomorphic to the submodule $U_{K}^{1-\iota}$ of $U_{K}$. The intersection $\mu_{K} \cap U_{K}^{-}$ is taken inside $U_{K}$.

A diagram chase involving the exact sequence $0 \longrightarrow O_{K}^{*} \longrightarrow U_{K} \longrightarrow C_{K} \longrightarrow$ $C l_{K} \longrightarrow 0$ and the analogous sequence for $K^{+}$shows that there is an exact sequence [19]

$$
0 \longrightarrow \mu_{K}^{-} \longrightarrow U_{K}^{-} \longrightarrow C_{K}^{-} \longrightarrow C l_{K}^{-} \longrightarrow 0 .
$$

It is important to use the definition of the minus class group $C l_{K}^{-}$that we give here. Often the minus class group of an abelian number field $K$ is defined to be the kernel of the norm map $N: C l_{K} \longrightarrow C l_{K^{+}}$. The present definition differs at most in the 2-part. It has several advantages: as we will see below, it is easy to compute the Galois cohomology of $\mathrm{Cl}_{K}^{-}$; the results for the 2-part are very similar to the results for the odd parts. I don't know how to do the calculations using the other definition.

Another advantage over the usual definition is the following. It is easy to deduce the following formula for the order of $C l_{K}^{-}$from the usual class number formula:

$$
\# C l_{K}^{-}=\frac{2}{\left[\mu_{K}: \mu_{K}^{-}\right]} \# \mu_{K} \prod_{\chi \text { odd }}-\frac{1}{2} B_{1, \chi} .
$$

This formula does not involve the unit index " $Q_{K}$ " of Hasse [5, Ch.20], which is, in general, difficult to compute. This time there is the factor $2 /\left[\mu_{K}: \mu_{K}^{-}\right]$, which is either 1 or 2 , but this quantity is easy to compute; it captures, in some sense, only the easy aspects of the unit index $Q_{K}$ and its calculation is precisely the content of Hasse's Satz 22 in [5].

In this secton we fix a complex abelian number field $K$ with $G=\operatorname{Gal}(K / \mathbf{Q})$. Let $\pi$ be the 2-part of $G$ with fixed field $k=K^{\pi}$. We fix a non-trivial character $\chi$ of $G$ of odd order. We denote the fixed field of $K$ under $\iota$ by $K^{+}$. Note that $k \subset K^{+}$.

Theorem 3.1. Let $P \subset \pi$ be a 2-group that does not contain $\iota$ and let $E=K^{P}$. Let $E^{+}$be the fixed field of $E$ under $\iota$. If all primes $r$ that ramify in $E^{+} \subset K$ satisfy $\chi(r) \neq 1$, then

(i) $\mathrm{Cl}_{K}^{-}(\chi)$ is a cohomologically trivial $O_{\chi}[P]$-module;

(ii) the natural map $C l_{E}^{-}(\chi) \longrightarrow C l_{K}^{-}(\chi)^{P}$ is bijective and the norm map $N$ : $C l_{K}^{-}(\chi) \longrightarrow C l_{E}^{-}(\chi)$ is surjective.

Proof. Note that $\operatorname{Gal}\left(K / E^{+}\right) \cong P \times\{1, \iota\}$. The proof follows the pattern of the proof of Theorem 2.1.

(i) It suffices to show that $\widehat{H}^{q}\left(P, C l_{K}^{-}(\chi)\right)=0$ for all $q \in \mathbf{Z}$. Consider the exact sequence

$$
0 \longrightarrow \mu_{K}^{-} \longrightarrow U_{K}^{-} \longrightarrow C_{K}^{-} \longrightarrow C l_{K}^{-} \longrightarrow 0
$$

We show that the $\chi$-parts of the $P$-cohomology groups of the first three modules are trivial. Lemma 1.1 then implies that $\widehat{H}^{q}\left(P, C l_{K}^{-}(\chi)\right)=0$ for all $q \in \mathbf{Z}$.

Since $\chi$ has odd order, it acts trivially on the 2-part of $\mu_{K}^{-}$and therefore on its $P$-cohomology groups. This shows that $\widehat{H}^{q}\left(P, \mu_{K}^{-}\right)(\chi)=0$ for all $q \in \mathbf{Z}$. By global class field theory $\widehat{H}^{q}\left(P, C_{K}\right)$ and $\widehat{H}^{q}\left(P, C_{K^{+}}\right)$are isomorphic to $\widehat{H}^{q-2}(P, \mathbf{Z})$ and have therefore trivial $G$-action and, since $\chi \neq 1$, trivial $\chi$-parts. It follows that $\widehat{H}^{q}\left(P, C_{K^{-}}\right)(\chi)=0$ for all $q \in \mathbf{Z}$. 
By local class field theory and the fact that $\chi(r) \neq 1$ for the primes $r$ that ramify in $E \subset K$ and $E^{+} \subset K^{+}$we have that $\widehat{H}^{q}\left(P, U_{K}\right)$ and $\widehat{H}^{q}\left(P, U_{K^{+}}\right)$have trivial $\chi$-parts. The proofs are similar to the proof of part (i) of Theorem 2.1.

(ii) The natural map $C_{E}^{-} / N\left(C_{K}^{-}\right) \longrightarrow C l_{E}^{-} / N\left(C l_{K}^{-}\right)$is surjective. We saw already in the proof of part (i) that $C_{E}^{-} / N\left(C_{K}^{-}\right)=\widehat{H}^{0}\left(P, C_{K}^{-}\right)$has trivial $\chi$-part. Therefore the norm map $N: C l_{K}^{-}(\chi) \longrightarrow C l_{E}^{-}(\chi)$ is surjective. Note that we only used the fact that $\chi \neq 1$ to prove this.

To prove the second statement, we consider the following diagram:

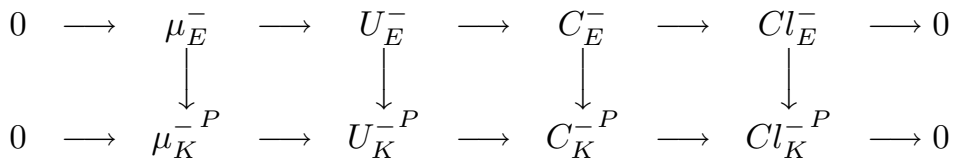

An easy diagram chase shows that the first three vertical arrows are injective and have cokernels with trivial $\chi$-parts. By the proof of part (i), the $P$-cohomology groups of each of the modules $\mu_{K}^{-}, U_{K}^{-}, C_{K}^{-}$and $C l_{K}^{-}$have trivial $\chi$-parts as well. This easily implies that the rightmost map induces an isomorphism $\mathrm{Cl}_{E}^{-}(\chi) \longrightarrow$ $C l_{K}^{-}(\chi)^{P}$ as required.

Theorem 3.2. If all primes $r$ that ramify in $k \subset K$ satisfy $\chi(r) \neq 1$, then

(i) there is an exact sequence

$$
0 \longrightarrow\left(O_{\chi}[\pi] /(1+\iota)\right)^{d} \stackrel{\Theta}{\longrightarrow}\left(O_{\chi}[\pi] /(1+\iota)\right)^{d} \longrightarrow C l_{K}^{-}(\chi) \longrightarrow 0 ;
$$

(ii) If, in addition, the prime 2 is not ramified in the field $K$, then

$$
\# C l_{K}^{-}(\chi)=O_{\chi} /\left(\prod_{\psi} \frac{1}{2} B_{1, \chi^{-1} \psi}\right),
$$

where the product runs over the odd characters $\psi$ of $G$ of 2-power order.

Proof. Choose $\sigma \in \pi$ so that $\langle\sigma\rangle$ is a direct summand of $\pi$ containing $\iota$. Let $2^{e}$ denote the order of $\sigma$ and let $P$ be a complement of $\langle\sigma\rangle$ in $\pi$ : we have $\pi=P \times\langle\sigma\rangle$. The eigenspace $C l_{K}^{-}(\chi)$ is a $O_{\chi}[\pi]$-module on which $\iota=\sigma^{2^{e-1}}$ acts as -1 . Therefore $C l_{K}^{-}(\chi)$ is a module over the ring $O_{\chi}[P \times\langle\sigma\rangle] /(1+\iota) \cong O_{\chi}\left[\zeta_{2^{e}}\right][P]$.

By Theorem 3.1, $C l_{K}^{-}(\chi)$ is a cohomologically trivial $P$-module. Let $O_{\chi}\left[\zeta_{2^{e}}\right][P]^{d}$ $\rightarrow C l_{K}^{-}(\chi)$ be a surjective $O_{\chi}\left[\zeta_{2^{e}}\right][P]$-homomorphism. The kernel is a cohomologically trivial torsion-free $O_{\chi}\left[\zeta_{2^{e}}\right][P]$-module. As in the proof of Theorem 2.3, we copy the proof of $[2, \mathrm{p} .113$, Thm.8] with $\mathbf{Z}$ replaced by the discrete valuation ring $O_{\chi}\left[\zeta_{2}\right]$. It follows that the kernel is projective and hence free over the local ring $O_{\chi}\left[\zeta_{2}\right][P]$. Since the quotient is finite, the kernel has rank $d$. This proves (i).

(ii) We proceed with induction with respect to the order of $\pi$. Since 2 is unramified we may apply C. Greither's Theorem [4, p.453, Thms. A and B] and we see that the result holds when $\pi$ is cyclic. Suppose $\pi$ is not cyclic. Writing $\pi=\langle\sigma\rangle \times P$ as in part (i), the group $P$ is not trivial. Let $\tau \in P$ be an element of order 2 . The fixed fields $K^{\tau}$ and $K^{\tau \iota}$ of $\tau$ and $\tau \iota$ are both complex abelian number fields containing $k$. The set of odd characters of $G$ is the disjoint union of the sets of odd characters of $\operatorname{Gal}\left(K^{\tau} / \mathbf{Q}\right)$ and $\operatorname{Gal}\left(K^{\tau \iota} / \mathbf{Q}\right)$.

By induction, the result holds for the fields $K^{\tau}$ and $K^{\tau \iota}$. By Theorem 3.1(i), $M=C l_{K}^{-}(\chi)$ is cohomologically trivial, both as a $\{1, \tau\}$-module and as a $\{1, \tau \iota\}$ module. Moreover, by part (ii) of that theorem, $(1+\tau) M$ and $(1+\tau \iota) M$ are isomorphic to the $\chi$-part of the 2-part of the minus class group of $K^{\tau}$ and $K^{\tau \iota}$ 
respectively. Since $\iota$ acts as -1 on $M$, it follows from the cohomological triviality of $M$ that $\# M=\#(1+\tau) M \cdot \#(1-\tau) M=\#(1+\tau) M \cdot \#(1+\tau \iota) M$. This proves (ii).

Finally we prove a sufficient condition for the eigenspace $C l_{K}^{-}(\chi)$ to be a cyclic $O_{\chi}[\pi] /(1+\iota)$-module.

Theorem 3.3. Suppose that all primes $r$ that ramify in $k \subset K$ satisfy $\chi(r) \neq 1$. If there exists an odd character $\varphi$ of odd conductor and of order $2^{k}$ for which each of the following two conditions hold:

$-\frac{1}{2} B_{1, \chi^{-1} \varphi}=\left(1-\zeta_{2^{k}}\right) u$ for some unit $u \in O_{\chi}\left[\zeta_{2^{e}}\right]^{*}$,

- $\chi(r) \neq 1$ for all primes $r$ dividing the conductor of $\varphi$, then $C l_{K}^{-}(\chi)$ is a cyclic $O_{\chi}[\pi] /(1+\iota)$-module.

Proof. Let $k_{\varphi}$ denote the composite field $k \mathbf{Q}^{\operatorname{ker} \varphi}$ and let $K_{\varphi}$ denote $K \mathbf{Q}^{\operatorname{ker} \varphi}$. Both fields $k_{\varphi} \subset K_{\varphi}$ are complex. Put $\pi^{\prime}=\operatorname{Gal}\left(K_{\varphi} / k\right)$ and $P=\operatorname{Gal}\left(K_{\varphi} / k_{\varphi}\right)$. We have that $\iota \notin P$.

Since 2 is not ramified, it follows from Greither's Theorem that the order of $C l_{k_{\varphi}}^{-}(\chi)$ is equal to the order of $O_{\chi} /\left(\operatorname{Norm}\left(\frac{1}{2} B_{1, \chi^{-1} \varphi}\right)\right)$. Here the Norm is the $O_{\chi}\left[\zeta_{2^{k}}\right] / O_{\chi}$-Norm. Since $\operatorname{Norm}\left(\frac{1}{2} B_{1, \chi^{-1} \varphi}\right)=\operatorname{Norm}\left(1-\zeta_{2^{k}}\right)=2$, we see that the order of $C l_{k_{\varphi}}^{-}(\chi)$ is equal to the order of the residue field of $O_{\chi}$. Therefore $C l_{k_{\varphi}}^{-}(\chi)$ is a cyclic Galois module. By Theorem 3.1, applied to $E=k_{\varphi} \subset K_{\varphi}$, the eigenspace $C l_{K_{\varphi}}^{-}(\chi)$ is a cohomologically trivial $P$-module and the $P$-norm map induces an isomorphism between $C l_{k_{\varphi}}^{-}(\chi)$ and $C l_{K_{\varphi}}^{-}(\chi)$ modulo the $P$-augmentation ideal. Therefore another application of Nakayama's Lemma implies that $C l_{K_{\varphi}}^{-}(\chi)$ is a cyclic $O_{\chi}[P]$-module and hence a cyclic $O_{\chi}\left[\pi^{\prime}\right] /(1+\iota)$-module. Therefore its quotient $C l_{K}^{-}(\chi)$ is a cyclic $O_{\chi}[\pi] /(1+\iota)$-module, as required.

If the group $\pi$ is cyclic, then $O_{\chi}[\pi] /(1+\iota) \cong O_{\chi}\left[\zeta_{2^{e}}\right]$ where $\# \pi=2^{e}$. Since the ring $O_{\chi}\left[\zeta_{2^{e}}\right]$ is a discrete valuation ring, the structure of finite modules over $O_{\chi}[\pi] /(1+\iota)$ is particularly simple.

Proposition 3.4. Suppose that $\pi$ is cyclic and that $C l_{K}^{-}(\chi)$ is cyclic over $O_{\chi}[\pi]$. If $\# C l_{K}^{-}(\chi)=2^{f t}$, where $2^{f}$ is the order of the residue field $O_{\chi} /(2)$, then there is an isomorphism of $O_{\chi}\left[\zeta_{2^{e}}\right]$-modules

$$
C l_{K}^{-}(\chi) \cong O_{\chi}\left[\zeta_{2^{e}}\right] /\left(\left(1-\zeta_{2^{e}}\right)^{t}\right)
$$

and there is an isomorphism of abelian groups

$$
C l_{K}^{-}(\chi) \cong\left(\mathbf{Z} / 2^{r} \mathbf{Z}\right)^{f\left(2^{e-1}-s\right)} \times\left(\mathbf{Z} / 2^{r+1} \mathbf{Z}\right)^{f s}
$$

where $r, s \in \mathbf{Z}$ are determined by $t=r 2^{e-1}+s$ and $0 \leq s<2^{e-1}$.

Proof. This follows from the fact that $O_{\chi}\left[\zeta_{2^{e}}\right]$ is a discrete valuation ring with uniformizing element $1-\zeta_{2}$.

\section{TABLES}

In this section we present the proof of Theorem II. An essential ingredient is the table of class numbers $h_{l}^{-}$given in the appendix. We briefly explain the notation. 
TABLE 4.1

\begin{tabular}{|l|l|l|l|l|l|}
\hline$l$ & & & $l$ & & \\
\hline 233 & $p_{14} \cdot p_{29}$ & $\mathrm{PM}$ & 419 & $p_{16} \cdot p_{30} \cdot p_{49}$ & PM, HtR \\
269 & $p_{16} \cdot p_{31}$ & $\mathrm{PM}$ & 433 & $p_{14} \cdot p_{34}$ & $\mathrm{PM}$ \\
317 & $p_{25} \cdot p_{49}$ & $\mathrm{HtR}$ & 439 & $p_{11} \cdot p_{21} \cdot p_{23} \cdot p_{24}$ & $\mathrm{PM}, \mathrm{PM}, \mathrm{PM}$ \\
337 & $p_{13} \cdot p_{15} \cdot p_{15}$ & $\mathrm{PM}, \mathrm{PM}$ & 449 & $p_{18} \cdot p_{84}$ & $\mathrm{PM}$ \\
359 & $p_{13} \cdot p_{30} \cdot p_{45}$ & $\mathrm{PM}, \mathrm{HtR}$ & 463 & $p_{18} \cdot p_{21} \cdot p_{25}$ & $\mathrm{PM}, \mathrm{BS}$ \\
379 & $p_{22} \cdot p_{24}$ & $\mathrm{BS}$ & 467 & $p_{19} \cdot p_{49} \cdot p_{55}$ & $\mathrm{PM}, \mathrm{AL}$ \\
383 & $p_{19} \cdot p_{24} \cdot p_{46}$ & $\mathrm{PM}, \mathrm{HtR}$ & 479 & $p_{20} \cdot p_{27} \cdot p_{70}$ & $\mathrm{PM}, \mathrm{AL}$ \\
389 & $p_{24} \cdot p_{60}$ & $\mathrm{AL}$ & 487 & $p_{30} \cdot p_{49}$ & HtR \\
397 & $p_{8} \cdot p_{26} \cdot p_{27}$ & $\mathrm{PM}, \mathrm{BS}$ & 499 & $p_{15} \cdot p_{18} \cdot p_{47}$ & $\mathrm{PM}, \mathrm{PM}$ \\
401 & $p_{16} \cdot p_{18} \cdot p_{31}$ & $\mathrm{PM}, \mathrm{PM}$ & 503 & $p_{12} \cdot p_{14} \cdot p_{112}$ & PM, PM \\
409 & $p_{12} \cdot p_{52}$ & $\mathrm{PM}$ & 509 & $p_{16} \cdot p_{28} \cdot p_{101}$ & $\mathrm{PM}, \mathrm{AL}$ \\
\hline
\end{tabular}

Let $l$ be an odd prime. We have $l-1=2^{e} \cdot m$ with $m$ odd. For every divisor $d$ of $l-1$ which itself is divisible by $2^{e}$ we define

$$
h_{l}^{-}(d)=\prod_{\operatorname{ord}(\chi)=d}-\frac{1}{2} B_{1, \chi}
$$

where the product runs over the characters $\chi:(\mathbf{Z} / l \mathbf{Z})^{*} \longrightarrow \mathbf{C}^{*}$ of order $d$; except when $d=l-1$, in which case we multiply this product by $l$, and when $d=2^{e}$, in which case we multiply it by 2 . In the rare occasion when $l-1$ is equal to $2^{e}$, the only possible value for $d$ is $l-1=2^{e}$ and we put

$$
h_{l}^{-}(d)=2 l \prod_{\operatorname{ord}(\chi)=d}-\frac{1}{2} B_{1, \chi} .
$$

This last case occurs only when $l$ is a Fermat prime i.e., when $l=3,5,17,257$, 65537 or has more than 2500000 decimal digits.

The numbers $h_{l}^{-}(d)$ are listed in the appendix. They are rational integers [5], [24] and they are related to the minus class number $h_{l}^{-}$by

$$
h_{l}^{-}=\# C l_{l}^{-}=\prod_{2^{e}|d| l-1} h_{l}^{-}(d) .
$$

In [15] D. H. Lehmer and J. M. Masley presented a table with the numbers $h_{l}^{-}(d)$ for $l \leq 509$. Of most of these numbers the complete prime factorization was given, but their table contains 22 unfactored composite numbers. These were factored by Peter Montgomery (PM), Bob Silverman (BS), Herman te Riele (HtR) and Arjen Lenstra (AL). The most laborious factorization, for $l=467$, was performed by Arjen Lenstra, who factored a 103 digit factor of $h_{467}^{-}$into a product of two primes of 49 and 55 digits respectively. We list the various contributions in Table 4.1. By $p_{n}$ we denote a prime factor of $n$ decimal digits. The order in which the initials are given corresponds to the order of the prime factors. In order to prove Theorem II and, at the same time, determine the structure of $\mathrm{Cl}_{l}^{-}$as an abelian group, we study the table of numbers $h_{l}^{-}(d)$ of the appendix. Clearly, if a prime $p$ divides the class number $h_{l}^{-}$exactly once, the $p$-part of $\mathrm{Cl}_{l}^{-}$is cyclic as a group and hence as a Galois module. This happens for most large prime divisors. All other cases are listed below. Tables 4.2, 4.3 and 4.4 contain the prime pairs $(p, l)$ with $l \leq 509$ for which $p^{2}$ divides $h_{l}^{-}$. We discuss each table in some detail. 
The class group $C l_{l}^{-}$is a product of its $p$-parts and each $p$-part is a product of eigenspaces $C l_{l}(\chi)$. The minus class group $C l_{l}^{-}$is a cyclic Galois module if and only if for each prime $p$, each eigenspace $C l_{l}^{-}(\chi)$ is cyclic over the local ring $O_{\chi}[\pi]$, where $\pi$ is the $p$-part of $G=\operatorname{Gal}\left(\mathbf{Q}\left(\zeta_{l}\right) / \mathbf{Q}\right)$.

TABle 4.2. Primes $p$ not dividing $l-1$

\begin{tabular}{|c|c|c|c|c|c|c|}
\hline$l$ & $p$ & $d$ & $f$ & $h_{l}(d)$ & class group & \\
\hline 41 & 11 & 40 & 2 & $11^{2}$ & $11 \times 11$ & \multirow{7}{*}{ Thm.2.3 with $r=283$} \\
\hline 131 & 3 & 26 & 3 & $3^{3}$ & $3 \times 3 \times 3$ & \\
\hline \multirow[t]{3}{*}{139} & 47 & 46 & 1 & $47^{2}$ & 2209 & \\
\hline & 277 & 46 & 1 & 277 & 277 & \\
\hline & & 138 & 1 & 277 & 277 & \\
\hline 149 & 3 & 4 & 2 & $3^{2}$ & $3 \times 3$ & \\
\hline 151 & 11 & 30 & 2 & $11^{2}$ & $11 \times 11$ & \\
\hline 157 & 157 & 156 & 1 & $157^{2}$ & $157 \times 157$ & \multirow[t]{3}{*}{ Thm.2.2 } \\
\hline \multirow[t]{2}{*}{211} & 281 & 14 & 1 & 281 & 281 & \\
\hline & & 70 & 1 & 281 & 281 & \\
\hline 227 & 2939 & 226 & 1 & $2939^{3}$ & $2939 \times 2939 \times 2939$ & \multirow[t]{4}{*}{ Thm.2.2 } \\
\hline 241 & 47 & 16 & 2 & $47^{2}$ & $47 \times 47$ & \\
\hline 277 & 47 & 276 & 2 & $47^{2}$ & $47 \times 47$ & \\
\hline \multirow[t]{2}{*}{281} & 11 & 40 & 2 & $11^{2}$ & $11 \times 11$ & \\
\hline & 41 & 40 & 1 & $41^{2}$ & 1681 & \multirow[t]{3}{*}{ Thm.2.3 with $r=83$} \\
\hline 293 & 3 & 4 & 2 & $3^{2}$ & $3 \times 3$ & \\
\hline 313 & 37 & 24 & 2 & $37^{2}$ & $37 \times 37$ & \\
\hline 337 & 17 & 16 & 1 & $17^{2}$ & $17 \times 17$ & Thm.2.2 \\
\hline 353 & 353 & 352 & 1 & $353^{2}$ & $353 \times 353$ & \multirow[t]{6}{*}{ Thm.2.2 } \\
\hline \multirow[t]{2}{*}{379} & 379 & 42 & 1 & 379 & 379 & \\
\hline & & 378 & 1 & 379 & 379 & \\
\hline 397 & 23 & 132 & 2 & $23^{2}$ & $23 \times 23$ & \\
\hline 401 & 41 & 80 & 2 & $41^{2}$ & $41 \times 41$ & \\
\hline 409 & 5 & 24 & 2 & $5^{2}$ & $5 \times 5$ & \\
\hline 419 & 3 & 2 & 1 & $3^{2}$ & 9 & Thm.2.3 with $r=7$ \\
\hline 443 & 3 & 26 & 3 & $3^{6}$ & $9 \times 9 \times 9$ & \multirow[t]{2}{*}{ Thm.2.3 with $r=7$} \\
\hline 457 & 5 & 24 & 2 & $5^{2}$ & $5 \times 5$ & \\
\hline 467 & 467 & 466 & 1 & $467^{2}$ & $467 \times 467$ & Thm.2.2 \\
\hline 479 & 5 & 2 & 1 & $5^{2}$ & 25 & \multirow[t]{3}{*}{ Thm.2.3 with $r=11$} \\
\hline \multirow[t]{3}{*}{487} & 7 & 2 & 1 & 7 & 7 & \\
\hline & & 6 & 1 & 7 & 7 & \\
\hline & 37 & 18 & 1 & $37^{2}$ & $37 \times 37$ & Thm.2.2 \\
\hline \multirow[t]{4}{*}{491} & 3 & 2 & 1 & $3^{2}$ & 9 & Thm.2.3 with $r=7$ \\
\hline & 11 & 10 & 1 & $11^{3}$ & $11 \times 121$ & Thm.2.2, Thm.2.3 with $r=23$ \\
\hline & 491 & 98 & 1 & 491 & 491 & \\
\hline & & 490 & 1 & $491^{2}$ & $491 \times 491$ & Thm.2.2 \\
\hline
\end{tabular}

In Table 4.2 we have listed all pairs $(p, l)$ for which $p$ is odd and $p^{2}$ divides $h_{l}^{-}$, but $p$ does not divide $l-1$. In this case the $p$-part $\pi$ of the Galois group of $\mathbf{Q}\left(\zeta_{l}\right)$ over $\mathbf{Q}$ is trivial and an eigenspace $C l_{l}(\chi)$ is cyclic as a Galois module if and only if it is a cyclic $O_{\chi}$-module. It turns out that in all cases every $C l_{l}(\chi)$ is cyclic as an $O_{\chi}$-module.

To explain the table, we first note that in the case $l=p$, the Teichmüller eigenspace $C l_{l}^{-}(\omega)$ is always trivial. Therefore we only have contributions for the 
TABLE 4.3. Odd primes $p$ dividing $l-1$

\begin{tabular}{|l|l|l|l|l|l|}
\hline$\ell$ & $p$ & $d$ & $h_{0}, h_{1}, \ldots$ & group & \\
\hline 31 & 3 & 2 & 3,3 & 9 & \\
11 & 7 & 2 & 7,7 & 49 & \\
101 & 5 & 4 & $5,25,25$ & $25 \times 125$ & Prop. $2.4, \lambda=2$ \\
131 & 5 & 2 & 5,5 & 25 & \\
137 & 17 & 8 & 17,17 & 289 & \\
157 & 3 & 2 & 3,3 & 9 & \\
181 & 5 & 12 & 13,13 & 169 & \\
199 & 3 & 2 & $9,3,3$ & 81 & \\
211 & 3 & 2 & 3,3 & 9 & \\
& 7 & 6 & 7,7 & 49 & \\
283 & 3 & 2 & 3,3 & 9 & \\
307 & 3 & 2 & $3,3,3$ & 27 & \\
331 & 3 & 2 & 3,9 & $3 \times 9$ & \\
& 3 & 10 & 81,81 & $9 \times 9 \times 9 \times 9=1$ \\
337 & 7 & 16 & 49,49 & $49 \times 49$ & Prop. $2.4, \lambda=1$ \\
367 & 3 & 2 & 9,3 & 27 & Prop.2.4, $\lambda=1$ \\
379 & 3 & 2 & $3,3,3,3$ & 81 & Prop.2.4, $\lambda=1$ \\
409 & 17 & 8 & 17,17 & 289 & \\
421 & 5 & 4 & 25,5 & 125 & \\
439 & 3 & 2 & 3,27 & $9 \times 9$ & \\
461 & 5 & 4 & 25,25 & $5 \times 125$ & Thm.2.3, $\theta=T^{2}-3 T-3$ \\
463 & 7 & 2 & 7,7 & 49 & \\
& 7 & 6 & 7,7 & 49 & \\
499 & 3 & 2 & 3,3 & 9 & \\
\hline
\end{tabular}

characters $\chi \neq \omega$. Let $d$ be a divisor of $l-1$ for which $p$ divides $h_{l}^{-}(d)$. Then for all characters $\chi$ of order $d$ the ring $O_{\chi}$ has a residue field with $p^{f}$ elements where $f$ is the order of $p$ modulo $d$. If $p^{f}$ happens to be the exact power of $p$ dividing $h_{l}^{-}(d)$, then it is clear that for exactly one character $\chi$ of order $d$ the eigenspace $C l_{l}^{-}(\chi)$ is isomorphic to $O_{\chi} /(2)$ while all others are trivial. These cases are listed without comment. In the remaining cases we apply the theorem of Mazur and Wiles which is the case with trivial $\pi$ of Theorem 2.2. If the precise power of $p$ dividing $h_{l}^{-}(d)$ is $p^{f a}$ and for precisely $a$ characters $\chi$ of order $d$ the generalized Bernoulli number $B_{1, \chi^{-1}}$ is divisible by $p$, then each eigenspace $C_{l}^{-}(\chi)$ is either isomorphic to $O_{\chi} /(2)$ or is zero. In particular, each $C l_{l}(\chi)$ is a cyclic Galois module. This happens in all but seven cases. In the remaining seven cases we use Theorem 2.3 and show that each eigenspace is a cyclic $O_{\chi}$ module by computing an additional Bernoulli number $B_{1, \chi^{-1} \varphi}$ where $\varphi$ is a suitable even character of order $p$ and conductor $r$.

In Table 4.3 we have listed all pairs $(p, l)$ with $p \neq 2$ dividing $l-1$. We'll see below that in this case the class number $h_{l}^{-}$is automatically divisible by $p^{2}$, so that Table 4.3 actually contains all pairs $(p, l)$ for which $p$ divides $\operatorname{gcd}\left(h_{l}^{-}, l-1\right)$. In order to explain the contents of the table, we fix $p$ and $l$ and we let $p^{e}$ be the exact power of $p$ dividing $l-1$.

If $d$ and $d^{\prime}$ are two divisors of $l-1$ that only differ by a power of $p$, then $B_{1, \varphi^{-1}} \equiv$ $B_{1, \varphi^{\prime-1}}$ modulo $\left(1-\zeta_{p^{e}}\right)$ for all characters $\varphi$ of order $d$ and $\varphi^{\prime}$ of order $d^{\prime}$. Therefore, as Lehmer observed [14, Thm.5], either both $h_{l}^{-}(d)$ and $h_{l}^{-}\left(d^{\prime}\right)$ are divisible by $p$ 
TABLE 4.4. $p=2$

\begin{tabular}{|l|l|l|l|l|l|l|l|}
\hline$\ell$ & $d$ & $\operatorname{ord}(\chi)$ & $2^{e}$ & $f$ & $h_{l}^{-}(d)$ & 2-class group & $r$ \\
\hline 29 & 28 & 7 & 4 & 3 & 8 & $2 \times 2 \times 2$ & \\
113 & 112 & 7 & 16 & 3 & 8 & $2 \times 2 \times 2$ & \\
163 & 6 & 3 & 2 & 2 & 4 & $2 \times 2$ & \\
197 & 28 & 7 & 4 & 3 & 8 & $2 \times 2 \times 2$ & 3 \\
239 & 14 & 7 & 2 & 3 & $8^{2}$ & $4 \times 4 \times 4$ & 3 \\
277 & 12 & 3 & 4 & 2 & $4^{2}$ & $2 \times 2 \times 2 \times 2$ & \\
311 & 62 & 31 & 2 & 5 & $32^{2}$ & $2 \times 2 \times 2 \times 2 \times 2 \times 2 \times 2 \times 2 \times 2 \times 2$ & \\
337 & 336 & 21 & 16 & 6 & 64 & $2 \times 2 \times 2 \times 2 \times 2 \times 2$ & \\
349 & 12 & 3 & 4 & 2 & $4^{2}$ & $2 \times 2 \times 2 \times 2$ & \\
373 & 124 & 31 & 4 & 5 & 32 & $2 \times 2 \times 2 \times 2 \times 2$ & \\
397 & 12 & 3 & 4 & 2 & $4^{3}$ & $4 \times 4 \times 2 \times 2$ & \\
421 & 60 & 15 & 4 & 4 & 16 & $2 \times 2 \times 2 \times 2$ & \\
463 & 14 & 7 & 2 & 3 & 8 & $2 \times 2 \times 2$ & \\
491 & 14 & 7 & 2 & 3 & $8^{2}$ & $2 \times 2 \times 2 \times 2 \times 2 \times 2$ & \\
\hline
\end{tabular}

or none is. For this reason we have ordered the class numbers as follows: for each divisor $d$ of $l-1$ which is itself not divisible by $p$ but for which $h_{l}^{-}(d)$ is divisible by $p$, we list, for $i=0,1, \ldots, e$ the $p$-part $h_{i}$ of $h_{l}^{-}\left(d p^{i}\right)$. By Lehmer's observation, each $h_{i}$ is divisible by $p$. We note in passing that this implies that $h_{l}^{-}$is divisible by $p^{2}$.

For each character $\chi$ of order $d$ the residue field of $O_{\chi}$ has order $p^{f}$ where $f$ is the order of $p$ modulo $d$. In all but one case either $h_{0}=p^{f}$ or $h_{1}=p^{f}$. In the latter case we have that, up to a unit, $B_{1, \chi^{-1} \psi}=1-\zeta_{p}$ for the characters $\psi$ of conductor $l$ and order $p$. In either case Theorem 2.3 applies and we see that $C l_{l}(\chi)$ is cyclic over $O_{\chi}[\pi]$. The only exception is $l=461$ with $p=5$. In this case $h_{0}=h_{1}=25$. In this case we have applied Theorem 2.3 with $\varphi$ a character of order 5 and conductor 11 . It turns out that in this exceptional case $C l_{l}(\chi)$ is a cyclic $O_{\chi}[\pi]$-module as well.

In most cases we can apply Theorem III and conclude that the eigenspace is a cyclic group. These cases are listed without comment. In the cases $(l, p)=(101,5)$, $(337,7),(461,5)$ and $(331,3)$ (the latter for $d=10)$ an application of Proposition 2.4 immediately gives the structure of $C l_{l}(\chi)$. Finally, in the cases $(l, p)=(439,3)$ and $(331,3)$ (the latter for $d=2$ ) we have explicitly computed the Stickelberger element $\theta$ and applied Theorem 2.3 directly.

Finally we discuss the contents of Table 4.4. Let $\chi$ be a character of $(\mathbf{Z} / l \mathbf{Z})^{*}$ of odd order. The 2-part of $C l_{l}^{-}$is a module over $O_{\chi}[\pi] /(1+\iota) \cong O_{\chi}\left[\zeta_{2^{e}}\right]$. Here $2^{e}$ is the exact power of 2 dividing $l-1$. It is well known that $C l_{l}^{-}(\chi)$ is trivial when $\chi=1$. This implies that the prime $p=2$ never divides $h_{l}^{-}$with multiplicity 1 . Therefore Table 4.4 actually contains all primes $l \leq 509$ for which $h_{l}^{-}$is even.

It turns out that $C l_{l}^{-}(\chi)$ is in all cases a cyclic Galois module. This follows from several applications of Theorem 3.3. In all but 4 cases we have that $\prod_{\psi} \frac{1}{2} B_{1, \chi^{-1} \psi}=$ $2 u$ for some unit $u \in O_{\chi}$. Here the product runs over the odd characters $\psi$ of 2 power order and conductor $l$. In this case $C l_{l}^{-}(\chi) \cong O_{\chi} /(2)$ which is a vector space of dimension $f$ over $\mathbf{F}_{2}$. Here $f$ is the degree of $\mathbf{F}_{2}\left(\zeta_{d}\right)$ over $\mathbf{F}_{2}$ and $d$ is the order of $\chi$. In the remaining cases we applied Theorem 3.3 with an odd quadratic character $\varphi$ of conductor $r$. Here $r \equiv 3(\bmod 4)$ is a prime for which $\chi(r) \neq 1$.

The structure of $\mathrm{Cl}_{l}^{-}(\chi)$ then follows easily from Theorem 3.4. 


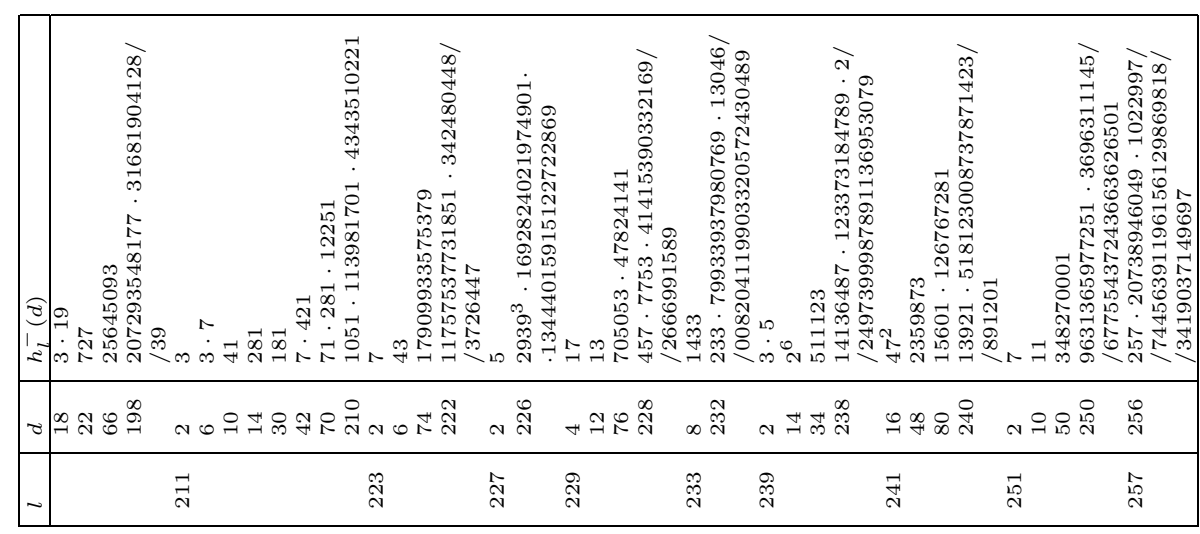

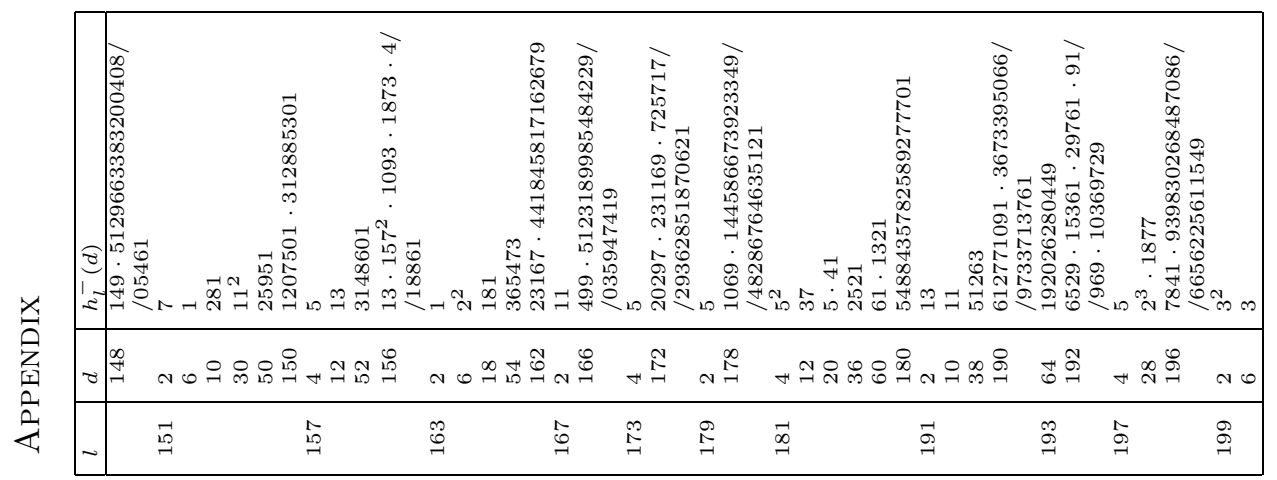

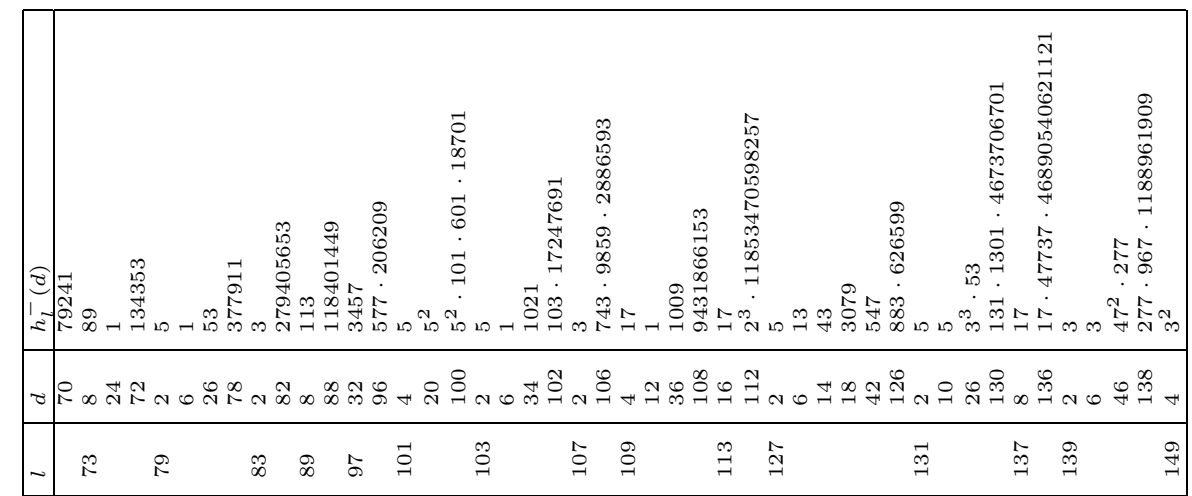

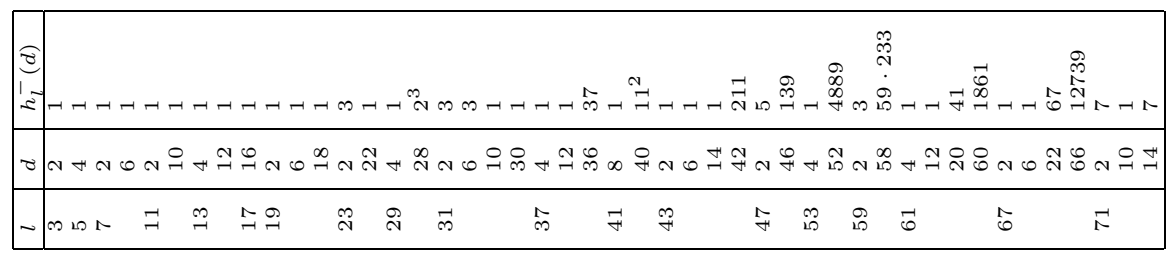




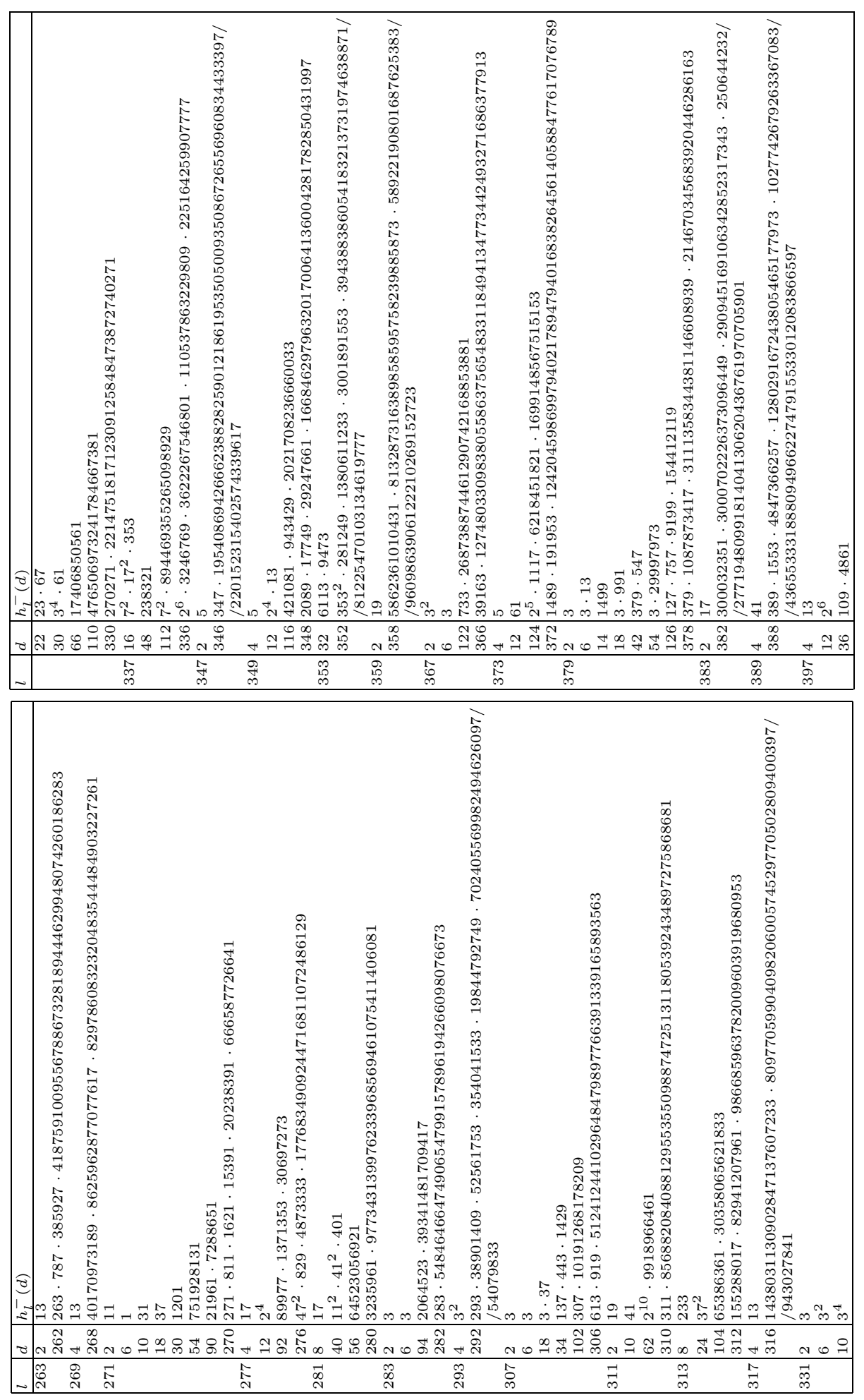


RENÉ SCHOOF

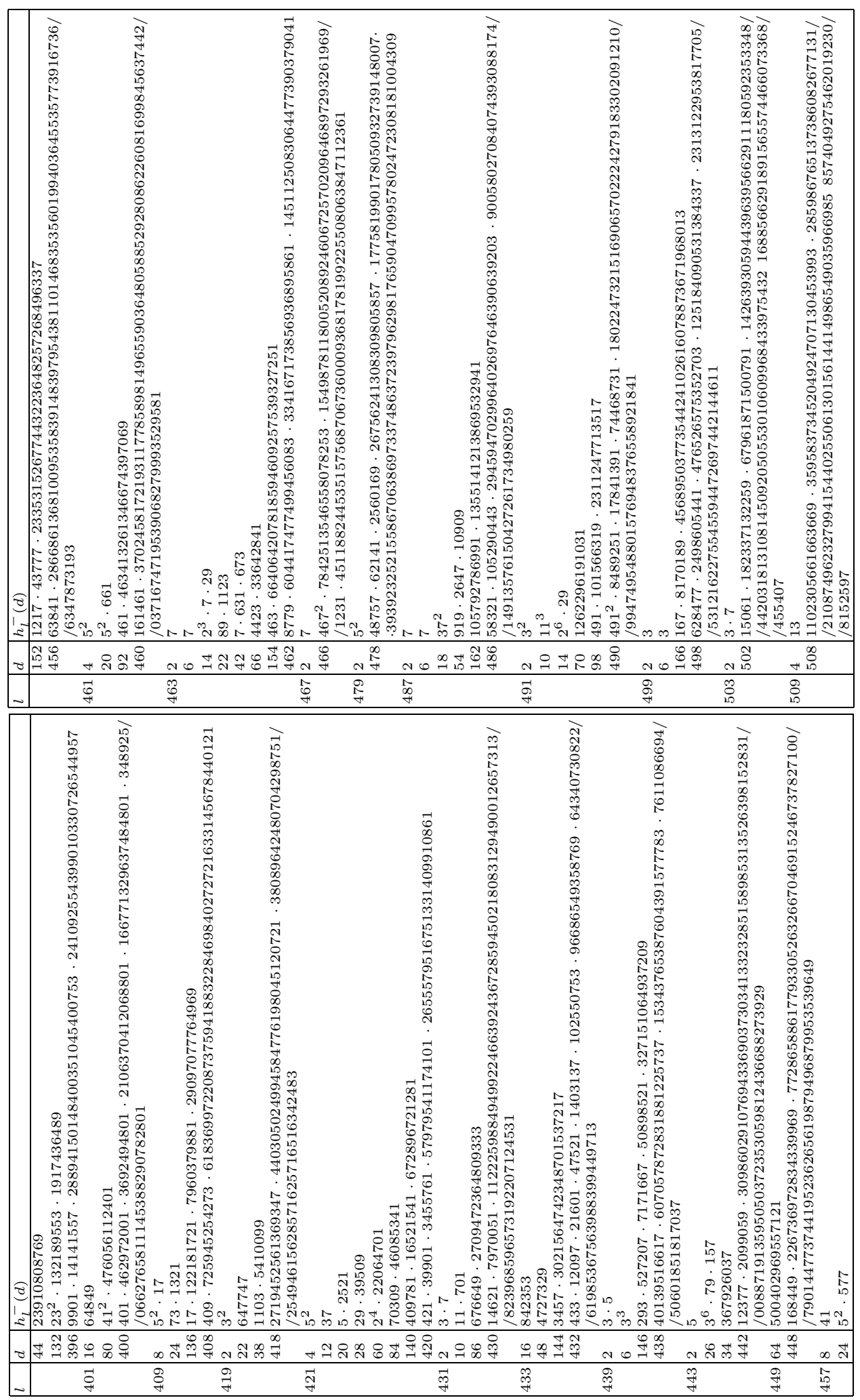




\section{REFERENCES}

[1] Bourbaki, N.: Éléments de Mathématique, Algèbre, Hermann, Paris 1970.

[2] Cassels, J.W.S and Fröhlich, A.: Algebraic Number Theory, Academic Press, London 1967. MR 35:6500

[3] Cornacchia, P.: Anderson's module and ideal class groups of abelian fields, J. Number Theory, to appear.

[4] Greither, C.: Class groups of abelian fields, and the main conjecture, Ann. de l'Institut Fourier, 42 (1992), 449-499. MR 93j:11071

[5] Hasse, H.: Über die Klassenzahl abelscher Zahlkörper, Akademie-Verlag, Berlin 1952. MR 14:141a

[6] Iwasawa, K.: A note on ideal class groups, Nagoya Math. J. 27, (1966), 239-247. MR 33:5603

[7] Kolyvagin, V.A.: Euler Systems, in: The Grothendieck Festschrift II, Prog. Math. 87, Birkhäuser, Boston 1990, 435-483. MR 92g:11109

[8] Kummer, E.E.: Collected papers, Vol.I, Springer-Verlag, Berlin 1975. MR 57:5650a

[9] Kummer, E.E.: Bestimmung der Anzahl nicht äquivalenter Classen für die aus $\lambda$ ten Wurzeln der Einheit gebildeten complexen Zahlen und die idealen Factoren derselben, J. für die reine und angewandte Math. 40, (1850), 93-116. (Coll.Papers 299-322)

[10] Kummer, E.E.: Mémoire sur la théorie des nombres complexes composés de racines de l'unité et de nombres entiers, J. de math. pures et appl. 16, (1851), 377-498. (Coll.Papers 363-484)

[11] Kummer, E.E.: Über die Irregularität von Determinanten, Monatsberichte der Kön. Preuß. Ak. der Wiss. zu Berlin, (1853), 194-200. (Coll.Papers 539-545)

[12] Kummer, E.E.: Über die aus 31sten Wurzeln der Einheit gebildeten complexen Zahlen, Monatsberichte der Kön. Preuß. Ak. der Wiss. zu Berlin, (1870), 755-766. (Coll.Papers 907918)

[13] Lang, S.: Cyclotomic fields, Graduate Texts in Math. 59, Springer-Verlag, New York 1978. MR 58:5578

[14] Lehmer, D.H.: Prime factors of cyclotomic class numbers, Math. Comp. 31, (1977), 599-607. MR 55:5576

[15] Lehmer, D.H. and Masley, J.: Table of the cyclotomic class numbers $h^{*}(p)$ and their factors for $200<p<521$, Math.Comp. 32, (1978), 577-582, with microfiche supplement. MR 58:16594a

[16] Mazur, B. and Wiles, A.: Class fields of abelian extensions of Q, Invent. Math. 76, (1984), 179-330. MR 85m:11069

[17] Perrin-Riou, B.: Travaux de Kolyvagin et Rubin, Séminaire Bourbaki 1989-1990, Exp. 717, Astérisque, 189-190, 69-106. MR 92f:11085

[18] Rubin, K.: Kolyvagin's system of Gauss sums, In: Arithmetic Algebraic Geometry, Texel 1989, Prog. Math. 89, Birkhäuser, Boston 1991. MR 92a:11121

[19] Schoof, R.: Cohomology of class groups of cyclotomic fields; an application to Morse-Smale diffeomorphisms, J. of Pure and Applied Algebra 53, (1988), 125-137. MR 89j:11111

[20] Schoof, R.: The structure of the minus class groups of abelian number fields, In: Goldstein. C.: Sém. de Théorie de Nombres, Paris 1988-1989, Birkhäuser, Boston 1990, 185-204. MR 92e: 11126

[21] Schoof, R.: Class numbers of $\mathbf{Q}(\cos (2 \pi / p))$, in preparation.

[22] Solomon, D.: On the class groups of imaginary abelian fields, Ann. Institut Fourier 40, (1990), 467-492. MR 92a:11133

[23] Van der Linden, F.: Class number computations of real abelian number fields, Math. Comp. 39, (1982), 693-707. MR 84e:12005

[24] Washington, L.C.: Introduction to cyclotomic fields, Graduate Texts in Math. 83, SpringerVerlag, New York 1982. MR 85g:11001

Dipartimento di Matematica, 2a Università di Roma "Tor Vergata", I-00133 Rome, ITALY

E-mail address: schoof@wins.uva.nl 